\title{
Charting the functional relevance of Broca's area for visual word recognition and picture naming in Dutch using fMRI-guided TMS
}

Citation for published version (APA):

Wheat, K. L., Cornelissen, P. L., Sack, A. T., Schuhmann, T., Goebel, R., \& Blomert, L. (2013). Charting the functional relevance of Broca's area for visual word recognition and picture naming in Dutch using fMRI-guided TMS. Brain and Language, 125(2), 223-230. https://doi.org/10.1016/j.bandl.2012.04.016

Document status and date:

Published: 01/01/2013

DOI:

10.1016/j.bandl.2012.04.016

Document Version:

Publisher's PDF, also known as Version of record

Document license:

Taverne

Please check the document version of this publication:

- A submitted manuscript is the version of the article upon submission and before peer-review. There can be important differences between the submitted version and the official published version of record.

People interested in the research are advised to contact the author for the final version of the publication, or visit the DOI to the publisher's website.

- The final author version and the galley proof are versions of the publication after peer review.

- The final published version features the final layout of the paper including the volume, issue and page numbers.

Link to publication

\footnotetext{
General rights rights.

- You may freely distribute the URL identifying the publication in the public portal. please follow below link for the End User Agreement:

www.umlib.nl/taverne-license

Take down policy

If you believe that this document breaches copyright please contact us at:

repository@maastrichtuniversity.nl

providing details and we will investigate your claim.
}

Copyright and moral rights for the publications made accessible in the public portal are retained by the authors and/or other copyright owners and it is a condition of accessing publications that users recognise and abide by the legal requirements associated with these

- Users may download and print one copy of any publication from the public portal for the purpose of private study or research.

- You may not further distribute the material or use it for any profit-making activity or commercial gain

If the publication is distributed under the terms of Article $25 \mathrm{fa}$ of the Dutch Copyright Act, indicated by the "Taverne" license above, 


\title{
Charting the functional relevance of Broca's area for visual word recognition and picture naming in Dutch using fMRI-guided TMS
}

\author{
Katherine L. Wheat ${ }^{\mathrm{a}, \mathrm{b}, \mathrm{c}, *}$, Piers L. Cornelissen ${ }^{\mathrm{c}}$, Alexander T. Sack ${ }^{\mathrm{a}, \mathrm{b}}$, Teresa Schuhmann ${ }^{\mathrm{a}, \mathrm{b}}$, \\ Rainer Goebel ${ }^{\mathrm{a}, \mathrm{b}}$, Leo Blomert ${ }^{\mathrm{a}, \mathrm{b}}$ \\ ${ }^{a}$ Department of Cognitive Neuroscience, Faculty of Psychology \& Neuroscience, Maastricht University, The Netherlands \\ ${ }^{\mathrm{b}}$ Maastricht Brain Imaging Centre, Maastricht, The Netherlands \\ ${ }^{\mathrm{c}}$ Department of Psychology, University of York, York YO10 5DD, UK
}

\section{A R T I C L E I N F O}

Article history:

Available online 23 May 2012

\section{Keywords:}

Transcranial magnetic stimulation

fMRI

Visual word recognition

Picture naming

Inferior frontal gyrus

Precentral gyrus

\begin{abstract}
A B S T R A C T
Magnetoencephalography (MEG) has shown pseudohomophone priming effects at Broca's area (specifically pars opercularis of left inferior frontal gyrus and precentral gyrus; LIFGpo/PCG) within $100 \mathrm{~ms}$ of viewing a word. This is consistent with Broca's area involvement in fast phonological access during visual word recognition. Here we used online transcranial magnetic stimulation (TMS) to investigate whether LIFGpo/PCG is necessary for (not just correlated with) visual word recognition by $\sim 100 \mathrm{~ms}$. Pulses were delivered to individually fMRI-defined LIFGpo/PCG in Dutch speakers 75-500 ms after stimulus onset during reading and picture naming. Reading and picture naming reactions times were significantly slower following pulses at 225-300 ms. Contrary to predictions, there was no disruption to reading for pulses before $225 \mathrm{~ms}$. This does not provide evidence in favour of a functional role for LIFGpo/PCG in reading before $225 \mathrm{~ms}$ in this case, but does extend previous findings in picture stimuli to written Dutch words.
\end{abstract}

(c) 2012 Elsevier Inc. All rights reserved.

\section{Introduction}

When studying visual word recognition, it is important to consider not just the input to and output from the reading system, but also to understand what is happening inside the "black box". Neuroimaging methods such as function magnetic resonance imaging (fMRI), magnetoencephalography (MEG) and electroencephalography (EEG) allow us to investigate the internal workings of the system by measuring correlations between overt behaviour and signals from cortical and subcortical structures with varying degrees of spatial and temporal resolution. A well accepted limitation of this approach, however, is that an observed correlation does not necessarily indicate that unperturbed activation within these brain regions is causally related to successfully performing the behavioural task under consideration (Pascual-Leone, Walsh, \& Rothwell, 2000). For example, it is difficult to control for strategic behaviours used by participants that are not necessary for a behavioural outcome per se, but which may nevertheless produce misleading correlations between behaviour and brain activity (Pascual-Leone, 1999; Pascual-Leone et al., 2000). While careful experimental design can minimise effects like these, thereby bol-

* Corresponding author. Address: Department of Cognitive Neuroscience, Faculty of Psychology \& Neuroscience, Maastricht University, P.O. Box 616, 6200 MD Maastricht, The Netherlands.

E-mail address: Katie.Wheat@maastrichtuniversity.nl (K.L. Wheat) stering the evidence for causality, nevertheless, neuroimaging methods alone can never be sufficient to make the case - they are inherently correlational. However, by disrupting normal brain function, transcranial magnetic stimulation (TMS) can create a transient 'virtual brain lesion' (Pascual-Leone, 1999; Walsh \& Rushworth, 1999). Therefore, by delivering TMS pulses appropriately in space and time (Walsh \& Rushworth, 1999), and comparing the consequences of the induced temporary lesions to appropriate controls (de Graaf \& Sack, 2011), researchers can infer whether activity in specific brain regions is indeed necessary for a given task (Pascual-Leone, 1999).

Recently, Cornelissen et al. (2009) reported an MEG study in which native English speakers carried out a passive viewing task. These authors found stronger responses to words than consonant strings, false fonts and faces in Broca's area within $100 \mathrm{~ms}$ of stimulus onset. Pammer et al. (2004) found activation in Broca's area at $\sim 130 \mathrm{~ms}$ during a modified lexical decision task. Wheat, Cornelissen, Frost, and Hansen (2010) used masked phonological priming of silent reading to demonstrate that Broca's area activity, specifically the pars opercularis of left inferior frontal gyrus and neighbouring precentral gyrus (LIFGpo/PCG), could be modulated by the phonological relationship between prime and target. This response was strongest at LIFGpo/PCG for primes and targets with complete phonological overlap (brein-BRAIN), within $100 \mathrm{~ms}$ of viewing a word. Together, these results suggest that Broca's area may be involved in rapid phonological access during visual word 
recognition. Further support for this hypothesis comes from Ashby, Sanders, and Kingston (2009), who recorded EEG as participants read targets with voiced and unvoiced final consonants (e.g., fad, fat). Targets were primed by pseudowords that were incongruent or congruent in voicing and vowel duration (e.g., fap, faz). Phonological feature congruency between prime-target pairs modulated ERPs by $80 \mathrm{~ms}$, indicating that sub-phonemic features can be activated rapidly during word recognition.

The fact that these studies show Broca's area activation within the first $\sim 100 \mathrm{~ms}$ of viewing a word and that this can be modulated by phonological relationship provides support that the activity seen relates to fast phonological access. In these MEG studies (Cornelissen et al., 2009; Pammer et al., 2004; Wheat et al., 2010), this frontal region of activity also appears in isolation, before activity is seen to spread forward from visual cortex across other putative nodes of the reading network that have been shown in fMRI (e.g. left mid fusiform gyrus, superior temporal gyrus, middle temporal gyrus, temporal pole, anterior IFG; Jobard, Crivello, \& TzourioMazoyer, 2003; Vigneau, Jobard, Mazoyer, \& Tzourio-Mazoyer, 2005), further suggesting that the phonological access thereby revealed may be necessary for encoding the printed words. Moreover, Wheat et al. (2010) demonstrated that their phonological priming at LIFGpo/PCG is not likely to be due to any strategic behaviour because the participants were not aware of the primes and therefore could not modify their behaviour based on predictions about prime-target relationship. However, although this evidence provides a strong case for fast phonological access that is necessary for encoding, as these techniques cannot demonstrate a causal relationship between brain signals and behaviour, not all alternatives can be discounted. For example, phonologically related activity could indicate a persistent neural association between orthography and phonology that is left over from learning to read - such as when using letter-sound knowledge to sound out a new word - but that no longer plays a part in visual word recognition (Whitney \& Cornelissen, 2005). Alternatively, the activity could relate to conflict resolution mechanisms such as those described by January, Trueswell, and Thompson-Schill (2009). This would be particularly relevant for phonological priming, where there is a potential conflict to resolve between different orthographic representations activating the same phonological representation (brein-BRAIN) versus two different orthographic and phonological representations (broin-BRAIN). Therefore, here we use TMS to chart the time points of the functional necessity of early LIFGpo/PCG activity for successful single word reading.

In order to demonstrate that TMS has had an effect on visual word recognition at all, it is necessary to show a change in an appropriate behavioural measure, such as vocal reaction time (VRT). However, a change in VRT in response to a written word, when a TMS pulse is delivered at a certain time to a specific stimulation site, would not necessarily show that the site was involved in word identification or encoding at that time. This is because an effect on VRT could equally be caused by a disruption to the speech output processes that are also necessary for successful reading aloud but that are not specifically related to the early encoding processes of word identification. It is therefore important to have an appropriate control condition that allows us to make the distinction between the encoding processes that lead to successful visual word recognition versus any speech output components that lead to successful naming and so will also have an effect on the vocal reaction time.

Schuhmann, Schiller, Goebel, and Sack (2009) used TMS to show that Broca's area is functionally involved in picture naming at around $300 \mathrm{~ms}$ after a target was presented. Dutch participants were shown pictures of monosyllabic Dutch nouns and asked to name them aloud. Using an online event-related triple-pulse TMS paradigm, these authors applied TMS to Broca's area between 150 and $575 \mathrm{~ms}$ following picture presentation. Vocal reaction times were slowest for pulses delivered around $300 \mathrm{~ms}$. Schuhmann et al. interpret this as a disruption to syllabification in line with the model of speech production presented by Indefrey and Levelt (2004). Syllabification is defined by Indefrey and Levelt as an abstract segmental representation that takes place prior to the activation of articulatory motor representations that are required for speech output, and occurs by around $330 \mathrm{~ms}$ after viewing a picture in their model. In a similar triple-pulse TMS design, Schuhmann, Schiller, Goebel, and Sack (2011) stimulated the posterior part of left superior temporal gyrus (Wernicke's area), posterior Broca's area (in the region of pars opercularis), and the midsection of the left middle temporal gyrus. Triple-pulse TMS stimulation of each site (in separate sessions) between 150 and $575 \mathrm{~ms}$ following picture presentation replicated the finding that Broca's area is critical for picture naming at around $300 \mathrm{~ms}$. Stimulation to Wernicke's area disrupted picture naming for pulses delivered around $400 \mathrm{~ms}$. Surprisingly, (based on Indefrey and Levelt's model) stimulation of middle temporal gyrus produced significantly slower VRTs when pulses were delivered at around $225 \mathrm{~ms}$ and again at around $400 \mathrm{~ms}$.

Thus, there is converging evidence that posterior Broca's area is functionally relevant for picture naming at around $300 \mathrm{~ms}$, although it is not yet clear precisely what function this region performs. Furthermore, on the basis of Schuhmann et al. (2009, 2011) and Vihla, Laine, and Salmelin (2006), it was not expected that this region would be involved in picture naming prior to $\sim 300 \mathrm{~ms}$. Though it should be noted that Schuhmann et al.'s $(2009,2011)$ earliest pulse trains started at $150 \mathrm{~ms}$ after stimulus onset, meaning that Broca's involvement prior to $150 \mathrm{~ms}$ cannot be ruled out for these studies.

Picture naming offers a useful control condition for comparison with reading aloud. The processes we are particularly interested in disrupting with TMS are the early encoding processes, such as the mapping of orthographic to phonological representations, which may occur at LIFGpo/PCG within the first $100 \mathrm{~ms}$. This type of phonological access may be required for visual word recognition, but would not be required for picture recognition. However, in an overt naming task, both reading aloud and picture naming would require articulatory-motor representations for speech output. Therefore, by comparing the effect of TMS to LIFGpo/PCG on picture naming and single word reading, we can dissociate between early encoding process and later speech output processes. TMS pulses delivered to the target site early during phonological encoding processes would affect just word reading (seen as a change in reaction time) but pulses delivered later during any articulatory motor output phase of processing would disrupt both word reading and picture naming.

Here, we extended the naming paradigm of Schumann et al. (2009) to include written words as well as pictures. Replication of Schuhmann et al.'s paradigm also allowed us to define the stimulation target region functionally rather than anatomically. In the Schuhmann et al. (2009) study, LIFGpo was specified in each participant individually based on their structural anatomy. While this does account for the anatomical variation between participants, it does not take into account the known functional variation of this region (e.g., Derrfuss, Brass, von Cramon, Lohmann, \& Amunts, 2009). Therefore, in the current study, we improved on Schuhmann et al.'s design by using an fMRI localiser that allows us to identify a phonologically relevant peak of activity in LIFGpo/PCG for each participant and to stimulate this site precisely. fMRI-guided TMS was shown by Sack et al. (2009) to be a more powerful localisation technique than using structural MRI data alone.

In sum, this research was designed to address the hypothesis that early LIFGpo/PCG activity seen at $\sim 100$ ms in MEG studies is functionally necessary for normal single word reading. We stimulated individually fMRI-defined LIFGpo/PCG target sites during word reading and picture naming and used reaction times as a measure of successful task performance. Slowed reaction times 
would be expected if intact neural processing at the stimulation site is necessary for the current task at the time of pulse delivery. For picture naming, we expected to replicate slowed reaction times for pulse delivery at $300 \mathrm{~ms}$ (Schuhmann et al., 2009) and to show no effect of TMS pulses delivered earlier than $300 \mathrm{~ms}$. In line with previous findings for written English (Ashby et al., 2009; Cornelissen et al., 2009; Pammer et al., 2004; Wheat et al., 2010), we predicted slowed reaction times for word reading when the TMS pulse was delivered around $100 \mathrm{~ms}$ after target presentation. This early pulse delivery was expected to coincide with fast access to phonology which is required for encoding written words but not picture stimuli. The $300 \mathrm{~ms}$ pulse delivery was expected to coincide with processes relevant to speech output, which would be required for both reading aloud and picture naming.

\section{Method}

\subsection{Participants}

Participants were 12 right-handed, native Dutch speakers, with no history of reading disorder, 2 of whom were male (Mean age $=21.5 ; S D=1.83$ ). Of these participants, 10 (2 male) subsequently underwent TMS (Mean age $=20.85 ; S D=1.46$ ). All had normal or corrected-to-normal vision and had no history of neurological or psychiatric disorders. The experiment was approved by the local medical-ethical committee, written informed consent was obtained before participation. Participants were screened for TMS experimentation safety by an independent medical supervisor, and were compensated with vouchers worth $€ 40$.

\subsection{Stimulus materials}

For both experiments, Presentation (Neurobehavioral Systems, Inc., Albany, NY) was used for to control stimulus presentation and record participants' responses.

Stimuli for the TMS experiment consisted of 30 high frequency, monosyllabic Dutch nouns (CELEX INL corpus) and 30 line drawings of the objects that the same Dutch nouns represent (adapted from Snodgrass \& Vanderwart, 1980). For the fMRI experiment, two additional stimulus categories were 30 pseudowords created from the real Dutch words by replacing one or two letters, and a black and white square checkerboard stimulus. The properties of these stimuli are given in Table 1 and examples in Fig. 1. Stimuli were always presented as white on a black background.

\section{3. fMRI design and procedure}

A blocked design was employed for the fMRI localiser. This ensured maximum power to localise activity related to each target category. A total of 240 trials, 60 per condition, were assigned to 3 functional acquisition runs containing 8 target blocks each. The 8 blocks, presented in a pseudorandom order, each consisted of 10 trials of a particular category. A trial comprised $600 \mathrm{~ms}$ blank screen followed by $1000 \mathrm{~ms}$ target, resulting in $16 \mathrm{~s}$ blocks. Target blocks were separated by a $16 \mathrm{~s}$ fixation screen. Each run started

Table 1

Means and standard deviations of stimulus properties.

\begin{tabular}{llll}
\hline Type & $\begin{array}{l}\text { Mean string } \\
\text { length }\end{array}$ & $\begin{array}{l}\text { Mean frequency per } \\
\text { million (CELEX INL) }\end{array}$ & $\begin{array}{l}\text { Mean horizontal } \\
\text { visual angle }\left(^{\circ}\right)\end{array}$ \\
\hline Dutch word & $3.8(0.71)$ & $69.30(105.98)$ & 2.2 \\
Pseudoword & $3.8(0.71)$ & - & 2.2 \\
Line drawing & - & $69.30(105.98)$ & 2.6 \\
Checkerboard & - & - & 2.6 \\
\hline
\end{tabular}
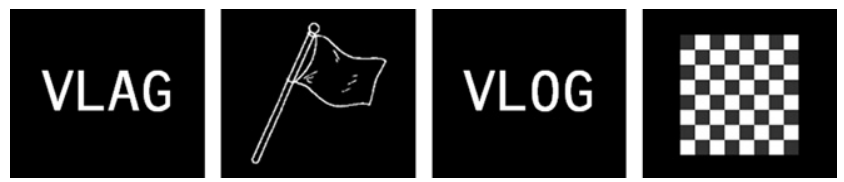

Fig. 1. Stimulus examples. The Dutch word 'vlag' (flag) was transformed into the nonword 'vlog' for the pseudoword condition.

with visual instruction followed by fixation. The duration of a run was $4.5 \mathrm{~min}$, resulting in a total duration of $13.5 \mathrm{~min}$. Participants were instructed to attend the fixation cross and to silently read or name the stimuli. Participants viewed all picture stimuli twice before the scan. They were instructed to read the stimuli out loud on the first presentation, in order to learn them, and to practise silent naming without any lips movements on the second presentation. The checkerboard was trained with the name "patroon" (pattern).

\subsection{TMS design and procedure}

An event-related single-pulse TMS (spTMS) design was employed in order to give the maximum temporal resolution. The experiment consisted of two sessions, one using real TMS, and one using the sham coil. Session order was counterbalanced across participants. A session consisted of 12 blocks, 6 of words and 6 of pictures, counterbalanced as to whether all words or all pictures were presented first.

Trial timings are depicted in Fig. 2. The (average) 35 trials per block had duration between 6850 and $8850 \mathrm{~ms}$, resulting in block lengths of $\sim 5$ min and session lengths of $1 \mathrm{~h}$. Pulses (1 per trial) were applied at $75,100,125,225,300$, or 500 ms post target onset. There was also an online control condition where no pulse was delivered. All stimuli appeared once under each pulse time (i.e., 7 times) in a within-subjects design. Trials were presented in a new random order for each participant.

Participants were instructed to read the stimuli aloud as quickly and accurately as possible. Responses were recorded using a digital microphone. TMS was applied using the MagProX100 stimulator (MagVenture A/S, Farum, Denmark) and a figure-of-eight coil (MC-B70, inner radius $10 \mathrm{~mm}$, outer radius $50 \mathrm{~mm}$ ). The maximum output of this coil and stimulator combination is approximately 1.9 Tesla and $150 \mathrm{~A} / \mu \mathrm{S}$. During sham stimulation, a specific figure-of-eight placebo coil (MC-P-B70) was used to reproduce the same acoustic stimulation as the active coil without inducing a magnetic field. The coils were manually held tangentially to the skull with the coil handle oriented perpendicular to the opercular part of the inferior frontal gyrus.

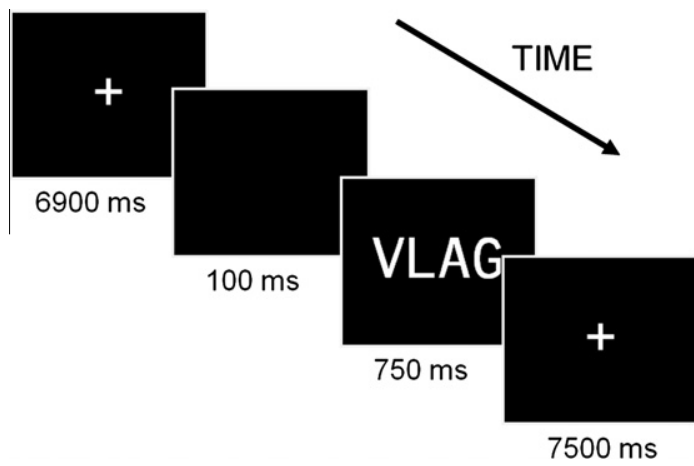

Fig. 2. Trial timings. Trials started with a fixation cross randomly jittered between 6000 and $8000 \mathrm{~ms}$ duration in steps of $100 \mathrm{~ms}$. Trials looped continuously. 
The target site was chosen based on each individual's fMRI data, by identifying a peak of frontal activity (for each individual separately) that fell within the approximate anatomical territory of pars opercularis of LIFG.

At the start of each TMS session, participants were coregistered to their structural brain imaging data using the BrainVoyager TMS Neuronavigator (Brain Innovation BV, Maastricht, the Netherlands). This allows for continuous online coil navigation to a target point on the cortical surface pre-defined based on the fMRI data. During the sessions, the proximity of the coil to the target point was continuously monitored and the coil position adjusted between trials when necessary.

Participants' resting motor threshold (RMT) was determined by positioning the coil over the hand area of the right motor cortex and testing for the stimulation level where hand movement could be observed on $50 \%$ pulses. The experimental stimulation level was then provisionally defined as $150 \%$ RMT, however, in practice, the level of facial muscle reactions generated by stimulating LIFGpo/ PCG limited the level of stimulation that could be applied to this site in most cases. Therefore, RMT was used as a starting point to find the maximum comfortable level of stimulation for each participant (up to a maximum of $200 \%$ RMT), which was tested by stimulating the LIFGpo/PCG target site while the participant read a practice list of words. The average stimulation level was $150 \%$ RMT (range 119-190\%).

\subsection{MRI data acquisition}

During each of the three functional runs, 150 functional volumes were acquired with a $\mathrm{T}_{2}{ }^{*}$ weighted echo-planar imaging (EPI) sequence (TE - echo time, $30 \mathrm{~ms}$, TR - repetition time, 2000 $\mathrm{ms}, \mathrm{FA}$ - flip angle, $90^{\circ}, 32$ slices with a thickness of $3.5 \mathrm{~mm}, 224$ $\mathrm{mm}$ FOV - field of view, with a $64 \times 64$ matrix resulting in $3.5 \times$ $3.5 \mathrm{~mm}$ in plane resolution). These were followed with a highresolution anatomical image, acquired using a magnetisationprepared rapid acquisition gradient echo (MPRAGE) sequence with a $1 \mathrm{~mm}$ isotropic resolution. This sequence consisted of $1921 \mathrm{~mm}$ sagittal slices with a matrix of 256 by 256 voxels (TR $=2250 \mathrm{~ms}$, $\mathrm{TE}=2.6 \mathrm{~ms}, \mathrm{FA}=9^{\circ}$ ). All MRI data were acquired in a 3-T magnetic resonance scanner (Siemens Allegra MR Tomograph; Siemens AG, Erlangen, Germany) with a volume head coil at the Faculty of Psychology and Neuroscience, Maastricht University, the Netherlands. Stimuli were displayed onto a frosted screen at the head end of the scanner bore, using an LCD projector (PLC-XT11-16, Sanyo North America Corporation, San Diego, CA). Subjects viewed the screen via an adjustable mirror mounted on the head coil.

\subsection{MRI analysis}

MRI data were analysed offline using BrainVoyager QX 2.3 (Goebel, Esposito, \& Formisano, 2006). The first four volumes of each functional run were excluded to allow for $\mathrm{T}_{1}$ saturation, permitting the $T_{2}$-weighted MR signal to stabilize. Pre-processing of the functional data consisted of slice scan time correction using cubic spline interpolation, 3D motion correction involving trilinear/ sinc interpolation, linear trend removal, and application of a $3 \mathrm{cy}-$ cles per time course $(2.83310-3 \mathrm{~Hz})$ high-pass filter. Functional runs were visually inspected to examine whether any subject moved excessively during data recording, which was not the case. The $2 \mathrm{D}$ functional runs were transformed into $3 \mathrm{D}$ volume time courses, aligned to the high-resolution anatomical volume. The high-resolution volumes were used for surface reconstruction of the left hemisphere of each participant. The surface reconstruction was performed in order to recover the exact spatial structure of the cortical sheet in order to improve the visualisation of the functional data in relation to the anatomy of each participant. This allowed for precise localisation of TMS stimulation using Neuronavigation.

For the group analyses, anatomical and functional data from each participant were transformed into Talairach standard space (Talairach \& Tournoux, 1988). Each participant's cortical surface (in Talairach space) was then aligned to create an average group surface reconstruction. Functional analyses were then performed using a random effects general linear model (RFX GLM) and the results overlaid on the group average surface reconstruction. The RFX GLM allows the generalisation of the group statistical inferences to the population level.

For the individual analyses, anatomical and functional data from each participant were aligned to AC-PC space (rotating the cerebrum into the anterior commissure-posterior commissure plane) without performing any spatial transformation. Functional analyses were performed for each participant separately using a fixed effects GLM and overlaid on their own surface reconstruction.

For both the RFX and fixed effects GLM, the fixation periods were entered into the model as the baseline. The predictor time courses in the model (for words, pseudowords, pictures and checkerboards) were adjusted to account for the delay in the hemodynamic response by convolution with a hemodynamic response function using the default settings in BrainVoyager QX 2.3 (Goebel et al., 2006).

\subsection{TMS response time analysis}

Responses were recorded using a microphone positioned directly in front of the participant. Sound level was calibrated individually prior to recording. Timings of voice key responses were logged by Presentation (Neurobehavioral Systems, Inc., Albany, $\mathrm{NY}$ ) and sound files of responses were recorded for offline analysis. All incorrect responses and voice key triggers other than verbal responses (such as coughs, hesitations and TMS pulse clicks) were removed prior to vocal reaction time (VRT) analysis. VRTs outside 4 $S D$ of the mean for a participant by type (word/picture), pulse time, and stimulation (TMS/sham) were treated as outlying data points and were excluded from further analysis.

\section{Results}

\section{1. $f M R I$}

RFX GLM group results shows regions of activity for each condition as compared to the passive baseline fixation condition. For all blocks other than fixation, participants were asked to name the stimuli silently in their head. As shown in Fig. 3, words, pseudowords and pictures show a peak of activity at the junction of the inferior frontal sulcus (IFS) and precentral sulcus (PCS), in other words, the border of LIFGpo and PCG. Note that this activity is thresholded in order to demonstrate the specific localisation of activity to IFS/PCS. At a lower threshold the pattern of BOLD responses can be seen to include cortical areas commonly activated by reading and visual word recognition tasks (Dehaene, Cohen, Sigman, \& Vinckier, 2005; Jobard et al., 2003; Vigneau et al., 2005). Also note that the small sample $(N=12)$ results in relatively low power; however, since the purpose of the fMRI experiment is to localise the activity in each individual, this group result is not critical to the main findings and is presented for illustrative purposes.

The contrast pseudowords $>$ words was expected to localise LIFGpo/PCG, based on an fMRI study by Wimmer et al. (2010) in German participants. Wimmer et al. described this activity as relating to the access of sublexical phonological segments and phonological productive processes. This contrast was therefore employed in 

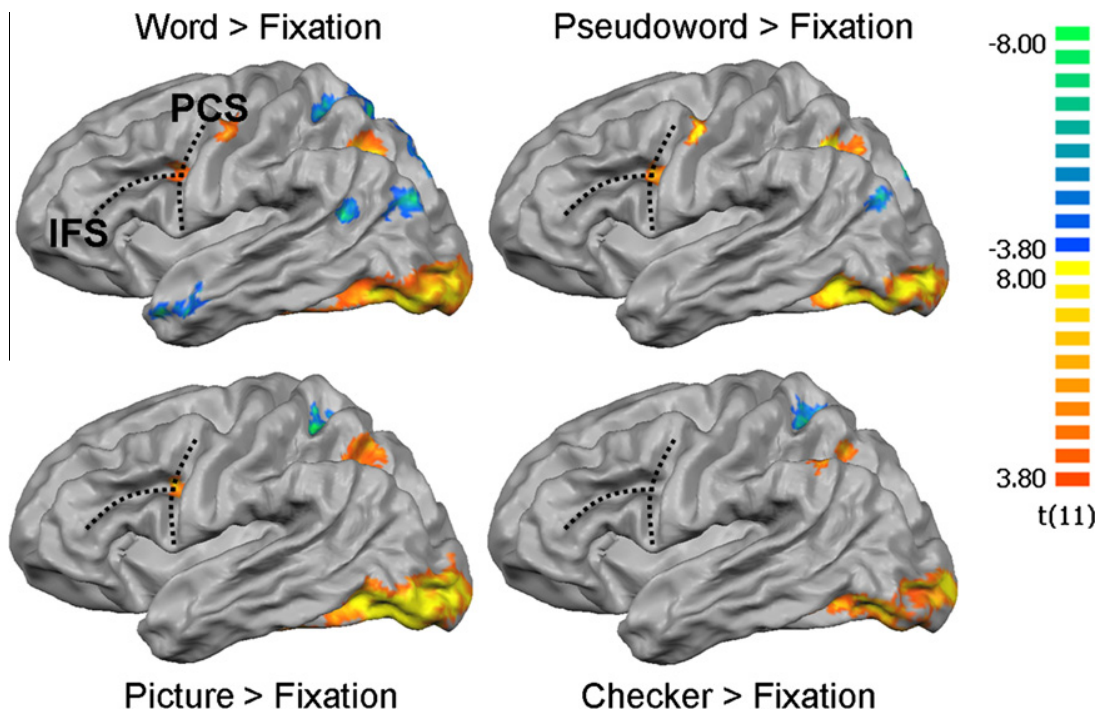

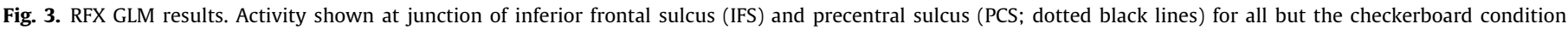
$(p<0.0005)$. Displayed on the average surface reconstruction of the 12 participants in Talairach space.

order to localise phonologically relevant areas of LIFGpo/PCG for each individual. Closely corresponding activity was found for all participants; the peak of this activity was then marked as the target point for TMS neuronavigation. Fig. 4a shows the variability of the individual target sites (red points) transformed into Talairach coordinates. The mean Talairach coordinate of the stimulation site was $-38,1,28$ (Mean Euclidean distance $=8.38 \mathrm{~mm}$ ), as shown in Fig. 4a (blue point). Fig. $4 \mathrm{~b}$ then shows the relationship between the average stimulation site and the peak of the group contrast pseudowords $>$ words at the border of LIFGpo/PCG and Fig. 4c shows the result of the contrast pseudowords $>$ words for one representative participant.

\subsection{TMS}

All incorrect, nonverbal and outlier responses were removed and data from one participant discarded due to the high number of missing or rejected trials. The no-pulse trials were also excluded from the analyses due to an oddball-like deviation from expectation when no pulse is delivered resulting in slower VRTs for these trials (see also De Graaf, Herring, \& Sack, 2011, for a similar occurrence).

\subsubsection{TMS induced changes in accuracy}

The mean error rates were calculated for each pulse time for both the real TMS and sham stimulation, for words and pictures separately. The mean error rates were: TMS pictures $=4.5 \%(S D$ $0.9)$; TMS words $=0.5 \%(S D 0.6)$; sham pictures $=3.1 \%(S D 0.7)$; sham words $=0.8 \%$ (SD 0.7).

A three-factor, repeated-measures ANOVA of number of errors was computed in SPSS. The within-subjects factors of TMS-sham (TMS vs. sham stimulation type), stimulus (word vs. picture stimuli), and pulse time (six levels) were entered into the model. The results showed a significant main effect of stimulus, $F(1,8)=$ 23.77, $p=.001, \eta_{p}^{2}=.75$, and a significant interaction between stimulus and TMS-sham, $F(1,8)=7.56, p=.03, \eta_{p}^{2}=.49$. No other effects were significant $(F<1.7, p>.17)$. These results demonstrate that participants made significantly more errors to the picture (a)

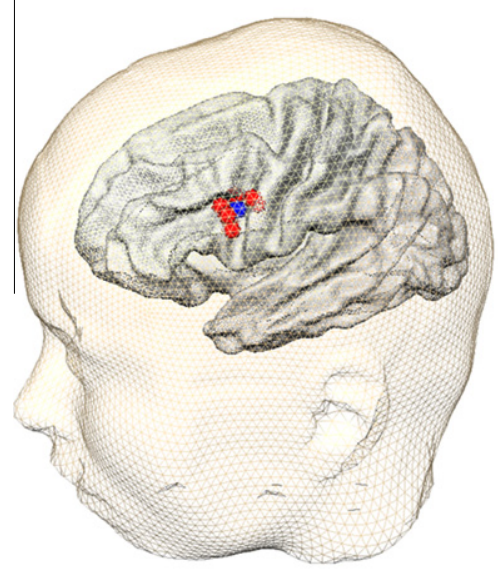

(b)

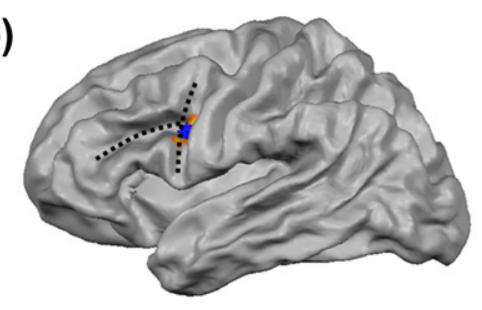

(c)

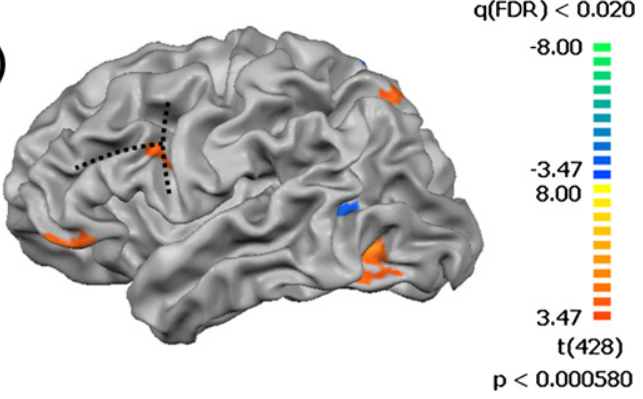

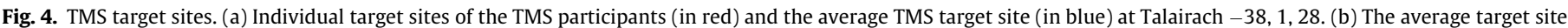

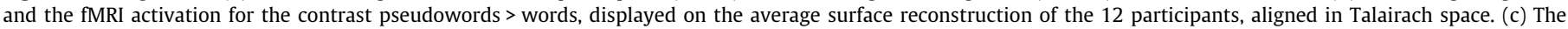
contrast pseudowords $>$ words for one representative participant, displayed on the individual surface reconstruction. 
stimuli, and that this difference was significantly greater under real TMS stimulation.

\subsubsection{TMS induced changes in VRT}

Mean VRTs were calculated for each pulse time for both stimulation and stimulus types and are plotted in Fig. 5. This figure shows a large difference between the sham and TMS VRTs for the no-pulse trials for words and pictures (34 ms and $21 \mathrm{~ms}$ difference, respectively). Due to these large differences, the no-pulse trials are excluded from all analyses.

A three-factor, repeated-measures ANOVA of VRTs was computed in SPSS. The within-subjects factors of TMS-sham (TMS vs. sham stimulation type), stimulus (word vs. picture stimuli), and pulse time (six levels) were entered into the model. The results showed significant main effects of stimulus, $F(1,8)=91.19$, $p<.001, \eta_{p}^{2}=.92$, and pulse time, $F(5,40)=4.83, p=.002, \eta_{p}^{2}=$ .38 , but not of TMS-sham, $F(1,8)=1.45, p=.26, \eta_{p}^{2}=.15$. There was also a significant TMS-sham $*$ pulse time interaction, $F(5$, $40)=2.69, p=.04, \eta_{p}^{2}=.25$. No other interactions were significant $(F<1.5, p>.26)$. These results show that pictures were named significantly slower than words, that the timing of pulse delivery affected VRTs, and that this was modulated by the type of stimulation applied (real vs. sham).

A separate two-factor, repeated-measures ANOVA of VRTs for TMS stimulation was computed for the factors stimulus and pulse time. The results showed a main effect of stimulus, $F(1,8)=75.98$, $p<.001, \eta_{p}^{2}=.91$, and of pulse time, $F(5,40)=3.36, p=.01, \eta_{p}^{2}=$ .30 , but no interaction, $F(5,40)=0.78, p=.57, \eta_{p}^{2}=.09$. This lack of interaction suggests that words and pictures are equivalently affected by TMS pulse delivered at different times. Planned polynomial contrasts revealed a significant quadratic effect of pulse time, $F(1,8), p=.008, \eta_{p}^{2}=.61$. Significant pairwise comparisons are indicated in Fig. 5 (corrected for multiple comparisons with Least Significant Difference).

A similar two-factor, repeated-measures ANOVA for sham stimulation was computed for the factors stimulus and pulse time. The

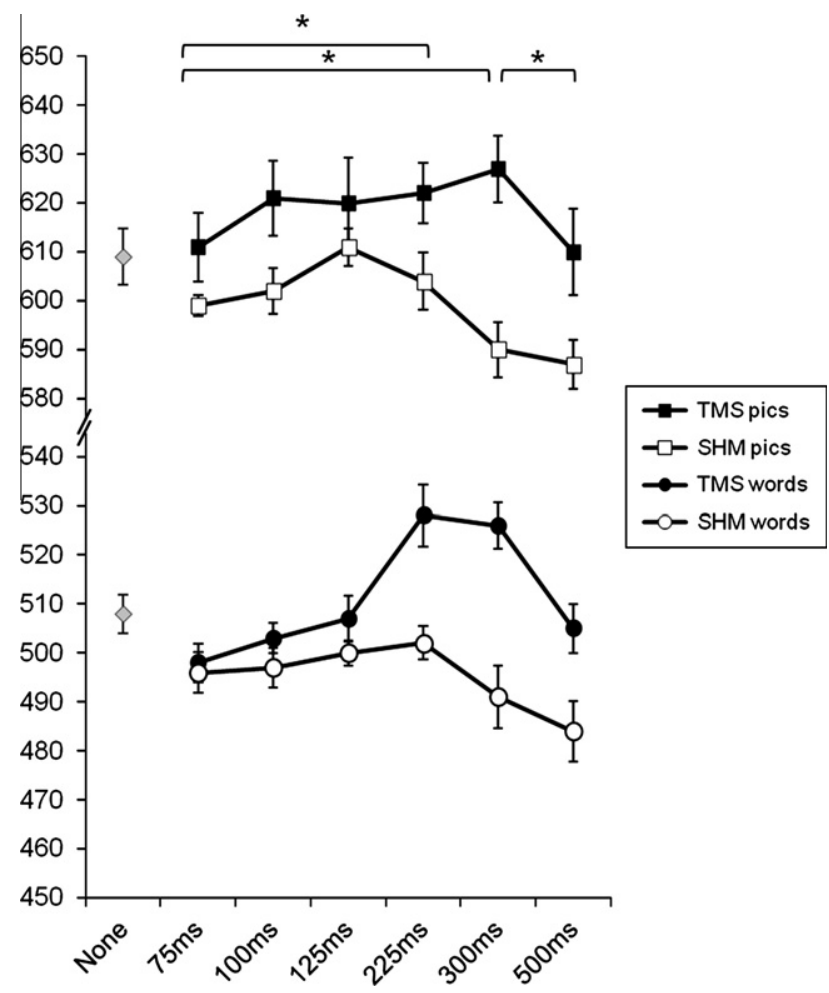

Fig. 5. Mean vocal reaction times. Reaction times for real and sham TMS for words and pictures. Pairwise comparisons for real TMS $\left({ }^{*} p<0.05\right)$. results showed a main effect of stimulus, $F(1,8)=60.36, p<.001$, $\eta_{p}^{2}=.88$, and of pulse time, $F(2.91,23.29)=3.96, p=.02, \eta_{p}^{2}=.33$ [corrected for a violation of sphericity, $\chi^{2}(14)=26.49, p<.05$ ], but no interaction, $F(5,40)=0.37, p=.87, \eta_{p}^{2}=.04$. Planned polynomial contrasts showed a small significant linear trend, $F(1,8)=$ 5.42, $p=.048, \eta_{p}^{2}=.40$.

\section{Discussion}

This study was designed to address whether LIFGpo/PCG activity observed within $\sim 100$ ms of viewing a word (Ashby et al., 2009; Cornelissen et al., 2009; Pammer et al., 2004; Wheat et al., 2010) is necessary for successful single word reading. In native Dutch speakers, we applied TMS to LIFGpo/PCG over a range of time delays with respect to stimulus onset and compared the impact of the TMS at each time delay on the vocal reaction times for word reading and picture naming. It was predicted that for picture naming, pulse delivery at $300 \mathrm{~ms}$ would result in slower VRTs (Schuhmann et al., 2009). For word reading, we predicted slower VRTs for TMS pulses delivered at around $100 \mathrm{~ms}$ as well as at $300 \mathrm{~ms}$.

As predicted, VRTs were slowest for picture naming when TMS pulses were delivered at $300 \mathrm{~ms}$ after viewing the picture. In addition, reaction times were slowed for pulse delivery at $225 \mathrm{~ms}$. Word reading was also disrupted by pulses delivered at 225$300 \mathrm{~ms}$ after word presentation. Contrary to expectations, there was no disruption to word reading for pulses delivered prior to $225 \mathrm{~ms}$. Pulses delivered between 75 and 125 ms produced no significant change to VRT or accuracy for words or for pictures.

With respect to picture naming, the results of the current study replicate those of Schuhmann et al. (2009) and are consistent with the interpretation that Broca's area is critically involved in picture naming, especially at $\sim 300 \mathrm{~ms}$ following stimulus picture presentation. This study also extends the work of Schuhmann et al. (2009) by showing that Broca's area activation at $\sim 225-300 \mathrm{~ms}$ is necessary for successful single word reading. In addition, the current study improved on Schuhmann et al.'s (2009) TMS target site selection procedure by using a prior fMRI task to locate the peak of phonologically related activity within LIFGpo/PCG for each participant.

In line with Indefrey and Levelt's (2004) account of speech production, the articulatory-motor syllabification process occurs by around $330 \mathrm{~ms}$ after viewing either a picture or a written word. This would coincide with the slowest reaction times seen here after TMS pulses were delivered at $\sim 300 \mathrm{~ms}$. Although our finding fits with Indefrey and Levelt's account of speech production, neither their model, nor the results presented here, explain how written words and pictures can activate syllabification processes at equivalent times, but then produce $\sim 100$ ms slower reaction times for pictures than for words. Our findings show the slowest mean response for picture naming at $300 \mathrm{~ms}$ and the slowest mean response for word reading at $225 \mathrm{~ms}$, though overall, VRTs at 225 and $300 \mathrm{~ms}$ are not significantly different from each other. It may be that pulses delivered just prior to $225 \mathrm{~ms}$ would have been even more effective at disrupting words, or pulses delivered just after $300 \mathrm{~ms}$ would have been more effective at disrupting pictures, thereby revealing a greater dissociation between the two processes, and relating more to the $\sim 100 \mathrm{~ms}$ difference in VRT. Indefrey (2011) recently updated the timing estimates for word production processes, including integrating Schuhmann et al.'s (2009) finding. Indefrey (2011) now suggests that abstract phonological syllabification processes do not take place until 355-455 ms, but suggests that due to the short stimuli and short naming latencies of Schuhmann et al. (2009), syllabification might be expected to occur earlier in this case. Indefrey (2011) also con- 
cedes (contrary to Indefrey \& Levelt, 2004) that subregions of IFG may have distinct roles for word production, including articulatory-motor planning aspects, rather than just abstract syllabification processes as previously argued.

Our results do not support the hypothesis that successful single word reading requires the involvement of LIFGpo/PCG within $\sim 100$ ms of word presentation. A number of interesting speculations follow from this, however further research in this direction is required before a more formal interpretation of the early role of LIFGpo/PCG can be put forward. The early activation of LIFGpo/PCG at $~ 100 \mathrm{~ms}$ has been shown using MEG in native English speakers, but should also be conducted in Dutch in order to investigate any possible differences in the early aspects of the visual word recognition network relating to the difference in the orthographic opacity of the two languages (e.g., Ziegler et al., 2010). Another important future direction is the replication of the current TMS experiment in native English speakers. These two experiments are important for the theoretical interpretation of the current results in relation to early visual word recognition processes. The difference in the role of LIFGpo/PCG for English and Dutch participants at around $\sim 100 \mathrm{~ms}$ that could be inferred from a comparison of the current TMS result to the previous MEG results (e.g., Wheat et al., 2010) cannot yet be confirmed.

There are some methodological aspects that should be considered when interpreting the results. First, the task design here necessitated using high frequency nouns due to the desire to use the same picture and word stimuli. For example, the identical pronunciation of the words and picture stimuli allowed us to control for any variation in voice key triggering by different initial phonemes. However, this may have had a detrimental effect on the expectation that native Dutch speakers will make use of any putative early phonological encoding at LIFGpo/PCG. Future experiments using low frequency words or nonwords are necessary to rule out an early role of LIFGpo/PCG under more difficult word recognition processes that may require a more complex process in order to solve the pronunciation or identity of the stimulus. Secondly, the choice of pulse strength and sequence may not have been optimal for disrupting any early activity at LIFGpo/PCG. The single pulse TMS paradigm allows precise temporal stimulation of a specific target site. While we can be confident (based on the fMRI-guided neuronavigation) that our target site was selected appropriately, we cannot rule out the possibility that reading relevant processes were indeed taking place at our target site that were not influenced by our particular choices for pulse sequence, strength and timing. We used a cluster of TMS pulse times around $100 \mathrm{~ms}$ in order to minimise the possibility that the processes of interest would take place at a short duration falling between our pulse times, however, it is still feasible that this has occurred. Future studies aiming to explore an early encoding role of LIFGpo/ PCG could use even more pulses delivered within the early period of interest, or use double- or triple-pulse paradigms with overlapping pulse delivery times. However, choice of pulse strength and sequence may be limited due to the fact that increasing pulse strength and number of pulses applied over LIFGpo/PCG will increase the strength of the facial muscle reactions experienced by participants (Wassermann, 1998). These muscle reactions may cause discomfort, and may also make it more difficult for participants to produce a vocal response and therefore have confounding effects on VRT.

\section{Conclusions}

In summary, this study replicated the findings of Schuhmann et al. (2009, 2011) that Broca's area (LIFGpo/PCG) is functionally relevant for picture naming from 225 to $300 \mathrm{~ms}$ after stimulus presentation in native Dutch speakers. Furthermore, this finding was extended to the reading aloud of real Dutch words, with VRT disruption occurring for words at the same pulse timings as for pictures. As predicted, picture naming was not affected by pulses delivered before $225 \mathrm{~ms}$. However, contrary to expectations, there was also no disruption to reading aloud within the $75-125 \mathrm{~ms}$ pulse timings for LIFGpo/PCG stimulation. Further work is necessary to investigate whether this finding generalises to Dutch low frequency or nonwords, and to a comparable experiment in native English speakers.

\section{Acknowledgments}

We thank our medical supervisor Cees van Leeuwen, and our independent physician Martin van Boxtel. Furthermore we thank Jeannette Boschma for her help in data acquisition and Felix Duecker for his assistance with figure preparation.

\section{References}

Ashby, J., Sanders, L. D., \& Kingston, J. (2009). Skilled readers begin processing subphonemic features by $80 \mathrm{~ms}$ during visual word recognition: Evidence from ERPs. Biological Psychology, 80, 84-94.

Cornelissen, P. L., Kringelbach, M. L., Ellis, A. W., Whitney, C., Holliday, I. E., \& Hansen, P. C. (2009). Activation of the left inferior frontal gyrus in the first $200 \mathrm{~ms}$ of reading: Evidence from magnetoencephalography (MEG). PLoS ONE, 4, e5359.

De Graaf, T. A., Herring, J., \& Sack, A. T. (2011). A chronometric exploration of highresolution-sensitive TMS masking. II. Effects on subjective and objective measures of vision. Experimental Brain Research, 209, 19-27.

De Graaf, T. A., \& Sack, A. T. (2011). Null results in TMS: From absence of evidence to evidence of absence. Neuroscience and Biobehavioral Reviews, 35, 871-877.

Dehaene, S., Cohen, L., Sigman, M., \& Vinckier, F. (2005). The neural code for written words: A proposal. Trends in Cognitive Sciences, 9, 335-341.

Derrfuss, J., Brass, M., von Cramon, D. Y., Lohmann, G., \& Amunts, K. (2009). Neural activations at the junction of the inferior frontal sulcus and the inferior precentral sulcus: Interindividual variability, reliability, and association with sulcal morphology. Human Brain Mapping, 30, 299-311.

Goebel, R. Esposito, F. \& Formisano, E. (2006). Analysis of functional image analysis contest (FIAC) data with Brainvoyager QX: From single-subject to cortically aligned group general linear model analysis and self-organizing group independent component analysis. Human Brain Mapping, 27, 392-401.

Indefrey, P. (2011). Frontiers: The spatial and temporal signatures of word production components: A critical update. Frontiers in Language Sciences. http://dx.doi.org/10.3389/fpsyg.2011.00255.

Indefrey, P., \& Levelt, W. J. M. (2004). The spatial and temporal signatures of word production components. Cognition, 92, 101-144.

January, D., Trueswell, J. C., \& Thompson-Schill, S. L. (2009). Co-localization of stroop and syntactic ambiguity resolution in Broca's area: Implications for the neural basis of sentence processing. Journal of Cognitive Neuroscience, 21, 2434-2444.

Jobard, G., Crivello, F., \& Tzourio-Mazoyer, N. (2003). Evaluation of the dual-route theory of reading: A metanalysis of 35 neuroimaging studies. Neurolmage, 20, 693-712.

Pammer, K., Hansen, P. C., Kringelbach, M. L., Holliday, I., Barnes, G., Hillebrand, A., et al. (2004). Visual word recognition: The first half second. NeuroImage, 22, 1819-1825.

Pascual-Leone, A. (1999). Transcranial magnetic stimulation: Studying the brainbehaviour relationship by induction of "virtual lesions". Philosophical Transactions of the Royal Society of London. Series B: Biological Sciences,, 354, 1229.

Pascual-Leone, A., Walsh, V., \& Rothwell, J. (2000). Transcranial magnetic stimulation in cognitive neuroscience-virtual lesion, chronometry, and functional connectivity. Current Opinion in Neurobiology, 10, 232-237.

Sack, A. T., Kadosh, R. C., Schuhmann, T., Moerel, M., Walsh, V., \& Goebel, R. (2009). Optimizing functional accuracy of TMS in cognitive studies: A comparison of methods. Journal of Cognitive Neuroscience, 21, 207-221.

Schuhmann, T., Schiller, N. O., Goebel, R., \& Sack, A. T. (2009). The temporal characteristics of functional activation in Broca's area during overt picture naming. Cortex, 45, 1111-1116.

Schuhmann, T., Schiller, N. O., Goebel, R., \& Sack, A. T. (2011). Speaking of which: Dissecting the neurocognitive network of language production in picture naming. Cerebral Cortex. http://dx.doi.org/10.1093/cercor/bhr155.

Snodgrass, J. G., \& Vanderwart, M. (1980). A standardised set of 260 pictures: Norms for name agreement, image agreement, familiarity, and visual complexity. Journal of Experimental Psychology: Human Learning and Memory, 6, 174-215.

Talairach, J., \& Tournoux, P. (1988). Co-planar stereotaxic atlas of the human brain. 3dimensional proportional system: An approach to cerebral imaging. New York: Thieme Medical Publishers.

Vigneau, M., Jobard, G., Mazoyer, B., \& Tzourio-Mazoyer, N. (2005). Word and nonword reading: What role for the visual word form area? NeuroImage, 27, 694-705. 
Vihla, M., Laine, M., \& Salmelin, R. (2006). Cortical dynamics of visual/semantic vs. phonological analysis in picture confrontation. Neuroimage, 33, 732-738.

Walsh, V., \& Rushworth, M. (1999). A primer of magnetic stimulation as a tool for neuropsychology. Neuropsychologia, 37, 125-136.

Wassermann, E. M. (1998). Risk and safety of repetitive transcranial magnetic stimulation: report and suggested guidelines from the International workshop on the safety of repetitive transcranial magnetic stimulation, June 5-7, 1996. Electroencephalography and Clinical Neurophysiology/Evoked Potentials Section, 108, 1-16.

Wheat, K. L., Cornelissen, P. L., Frost, S. J., \& Hansen, P. C. (2010). During visual word recognition phonology is accessed within $100 \mathrm{~ms}$ and may be mediated by a speech production code: Evidence from MEG. Journal of Neuroscience, 30, 5229-5233.

Whitney, C. S., \& Cornelissen, P. L. (2005). Letter-position encoding and dyslexia. Journal of Research in Reading, 28, 274-301.

Wimmer, H., Schurz, M., Sturm, D., Richlan, F., Klackl, J., Kronbichler, M., et al. (2010). A dual-route perspective on poor reading in a regular orthography: An fMRI study. Cortex, 46, 1284-1298.

Ziegler, J. C., Bertrand, D., Toth, D., Csepe, V., Reis, A., Faisca, L., et al. (2010) Orthographic depth and its impact on universal predictors of reading: A crosslanguage investigation. Psychological Science, 21, 551-559. 